\title{
Políticas lingüísticas europeas y españolas: el camino hacia el cambio en la educación terciaria
}

Concha Julián-De-VegA

Consejería de Educación en el Reino Unido e Irlanda/Universidad de Huelva

JAVIER ÁvILA-LÓPEZ

Universidad de Córdoba

Received: 23 $3^{\text {rd }}$ September 2017 / Accepted: $4^{\text {th }}$ April 2018

ISSN: $1697-7467$

\begin{abstract}
RESUMEN: La misión de la universidad es dotar al alumnado con recursos para el mercado de trabajo y dar formación en un mundo globalizado. Las lenguas y sus culturas son instrumentos esenciales en la sociedad multilingüe en la que desarrollamos nuestro potencial como ciudadanos. Las políticas lingüísticas salvaguardan y facilitan la igualdad de oportunidades en el mercado laboral y es por este papel central por lo que se convierten en objeto de estudio en contextos educativos. El Consejo de Europa, la Comisión Europea y el Centro de Lenguas de Graz en sus recomendaciones identifican al plurilingüismo como principio básico de las políticas lingüísticas y el enfoque AICLE como itinerario eficaz para alcanzar este objetivo. España acogió con gran entusiasmo este enfoque desde sus comienzos y ha llegado a ser enclave de referencia en su implantación. En la educación superior el desarrollo ha sido heterogéneo en primera instancia. Las políticas lingüísticas europeas llegan a la educación terciaria española de la mano de la implantación previa en educación primaria y secundaria, permitiendo completar el círculo de la oferta educativa multilingüe. Las recientes medidas tomadas por la CRUE y ACLES intentan establecer un marco común en la promoción del multilingüismo en la universidad española.

Palabras clave: Políticas lingüísticas, multilingüismo, plurilingüismo, AICLE, enseñanza superior.
\end{abstract}

\section{European and Spanish Language Policies: The Road to Change in Tertiary Education}

\begin{abstract}
The mission of universities is to equip students with skills for the labour market and provide training for our global world. Languages and cultures are key tools in our multilingual social reality where our potential as citizens of the world is developed. Thus, language policies safeguard and facilitate the equality of opportunities, and it is in this central role they become an object of study in educational contexts. The Council of Europe, the European Commission and the European Centre of Modern Languages (Graz) recommends multilingualism as a basic principle and a central objective of language policies, and advocates CLIL as the path to reach the goal. Spain embraced multilingual projects right from the start and has become a landmark in their implementation; however, in higher education, their development has been rather heterogeneous from the beginning. Multilingual language policies were first developed in primary and secondary education, paving the way for its further expansion to tertiary education and, therefore, closing the circle of multilingual provision. Recent measures issued by the Chancellors' Conference
\end{abstract}


and the Association of Higher Education Language Centres attempt to set the path for a common framework in the promotion of multilingualism in Spanish universities.

Keywords: Language policy, multilingualism, plurilingualism, CLIL, higher education.

\section{INTRODUCCIÓN}

Vivimos en un mundo en continuo cambio donde la comunicación entre culturas se hace esencial para las relaciones internacionales, la creación de riqueza, el empleo y la capacidad de movilidad individual. Los medios de comunicación, la movilidad y las nuevas tecnologías juegan un papel esencial en la interacción que se establece entre culturas donde las idiosincrasias nacionales y regionales pasan a un segundo plano y se vive una internacionalización que diluye las diferencias creando identidades más complejas. El multilingüismo ${ }^{1} \mathrm{y}$, por ende, la política adoptada por las naciones con respecto a las lenguas es fundamental para entender la complejidad de las sociedades que se están fraguando y, a la vez, comprender la importancia de ampliar los horizontes de los ciudadanos del siglo XXI. Por consiguiente, el multilingüismo y la multiculturalidad se pueden considerar como parte fundamental en la construcción de la realidad social. El aprendizaje de lenguas y de sus culturas se ha establecido como uno de los grandes desafíos que se ha planteado Europa y serán las decisiones de las políticas educativas en cada país las que hagan posible convertir este reto en una realidad.

La labor de la universidad no puede estar de espaldas a estos objetivos puesto que su misión principal es preparar a su alumnado para el mercado de trabajo y dar oportunidades de formación frente a esta necesidad de competencia lingüística y multicultural en un mundo globalizado. Los esfuerzos por la internacionalización de la enseñanza terciaria pautadas por la Declaración de Bolonia (European Ministers in charge of Higher Education, 1999) marcaron un antes y un después en las políticas lingüísticas universitarias. Este artículo trata de resumir los antecedentes que han señalado el camino a estas políticas y esto no se puede entender, en el caso de España y sus comunidades autónomas, sin exponer previamente la trayectoria de las decisiones europeas, a nivel supranacional, de las nacionales y de las regionales con respecto al aprendizaje de las lenguas y sus culturas en la enseñanza no universitaria. El acogimiento de este movimiento desde la escuela ha pautado el camino a la universidad española.

\section{Políticas lingüísticas en europa durante las Últimas déCadas}

La promoción de las lenguas ha estado siempre presente desde el nacimiento de la Comunidad Económica Europea, aunque no fuera de manera explícita. Al final de los años 80, el incremento de la globalización económica, la internacionalización de la ciencia y de la educación superior supuso la necesidad de políticas lingüísticas más explícitas. Además, el

1 Se utilizará el témino multilingüismo como la característica de algunas zonas geográficas o sociedades que utilizan diferentes lenguas, frente a plurilingüismo que se usará como la descripción del repertorio lingüístico de un individuo. En http://www.coe.int/t/DG4/linguistic/Division_EN.asp, consultado el 24 de febrero de 2015. 
desarrollo de las nuevas tecnologías y las nuevas expectativas del Mercado Único Europeo (1992) con las adscripciones de nuevos socios con diferentes lenguas y la movilidad en el mercado único de trabajo incrementaron la necesidad de considerar el multilingüismo como uno de los ejes para el desarrollo de la identidad europea.

El Tratado de Maastricht (1992) marcó un hito en las políticas lingüísticas porque, si bien consideraba que los estados miembros eran soberanos de los sistemas educativos, establecía medidas incentivadoras para la enseñanza de lenguas que respondían a la internacionalización y la necesidad de equipar a los jóvenes europeos para el mercado de trabajo y la movilidad laboral.

En 1995, la Comisión Europea establece el objetivo de que los ciudadanos europeos dominen tres lenguas comunitarias y subraya la necesidad de que los países miembros se aseguren de que el aprendizaje de lenguas no esté reservado a una élite sino que esté presente en los sistemas educativos de manera generalizada. En este momento, el multilingüismo aparece como un factor de la identidad y ciudadanía europea. En el año 2000, la Estrategia de Lisboa incluye explícitamente la necesidad de dominar tres lenguas comunitarias. A esto siguieron medidas para establecer un marco de acción como fue el lanzamiento del Sello Europeo o del Año Europeo de las Lenguas en el 2001, así como medidas para alcanzar una mayor coherencia a nivel europeo, tales como los indicadores comunes de evaluación, el Eurobarómetro de lenguas, intercambios de experiencias, la cooperación y la coordinación.

El Consejo de Barcelona en 2004 hace aún más explícita la necesidad del aprendizaje de lenguas con el logo: 1+2, una lengua materna más dos. A partir de aquí se estableció el Plan de Acción: Promoting Language Learning and Linguistic Diversity: An Action Plan 2004-2006, que pretendía unificar las actividades e iniciativas que se estaban desarrollando en los campos educativos y formativos y establecía medidas de cooperación entre estados, esto es, a nivel supranacional, así como una financiación para el desarrollo de materiales para la enseñanza de lenguas y para el asesoramiento de expertos en aprendizaje y evaluación de lenguas. En 2005, el Marco Estratégico del Multilingüismo unió iniciativas, incluyó la importancia de las lenguas minoritarias y puso de relieve la importancia del multilingüismo como una de las competencias que ayudaba al crecimiento económico, la competitividad, la cohesión social y el crecimiento del ciudadano europeo. Esta medida marcó definitivamente las políticas europeas con respecto a las lenguas como herramientas para el desarrollo de la Unión, la internacionalización de la educación, las negociaciones entre las prioridades supranacionales y las políticas nacionales educativas, y la inclusión de la política lingüística dentro de medidas económicas y sociales de carácter general.

Aparte de las acciones de la Unión Europea, el Consejo de Europa, dado su carácter de organización intergubernamental basada en la defensa de los principios democráticos e inclusivos, ha impulsado importantes medidas también a través de la División de Políticas Lingüísticas y del Centro Europeo de Lenguas Modernas (ECML) de Graz, donde los grupos de trabajo están formados por investigadores de las universidades europeas más importantes en el campo de la lingüística aplicada y de la educación. La Conferencia de Estrasburgo en 1997 puso especial énfasis en la importancia de la comunicación intercultural y el plurilingüismo como objetivos claves. El Año Internacional de las Lenguas (2001) y la publicación del Common European Framework of Reference for Languages: Learning, Teaching, Assessment (2001) marcaron un antes y un después en las políticas lingüísticas 
europeas. El Marco proporcionó coherencia apoyando la movilidad entre naciones, el entendimiento mutuo y la cooperación. Desde ese momento, el Consejo de Europa ha orientado sus actuaciones a una serie de áreas tomando como referencia este documento. Áreas que van desde el desarrollo de instrumentos para la planificación y la evaluación conjunta, así como el reconocimiento de cualificaciones a nivel europeo, la coordinación de las medidas lingüísticas y el desarrollo de prioridades e informes al respecto, y, por último, la expansión de estas políticas no sólo a las lenguas extranjeras sino a las lenguas maternas, las lenguas oficiales y otras lenguas. En 2018, se ha publicado un nuevo documento complementario: The CEFR Companion Volume with New Descriptors (2018), desarrollado por la División de Políticas Lingüísticas del Consejo de Europa.

Como se puede observar, el marco de políticas lingüísticas comunes proporcionó y sigue proporcionando un gran apoyo en el avance del desarrollo y conservación de lenguas en Europa. A esto se han unido también acciones prácticas que han calado en el ámbito local. Para ello, el Consejo de Europa ha estado promocionando grupos de investigación y talleres sobre diferentes temáticas relacionadas con las lenguas y, por su parte, la Unión Europea ha desarrollado programas educativos donde se ha dado apoyo a las políticas lingüísticas desarrolladas. Este es el caso de los programas Lingua, Erasmus, Socrates, Leonardo, Comenius, el Programa de Aprendizaje Permanente (Lifelong Learning Programme) (2007-2013) y el último Erasmus + (2014-2020). Todos ellos, bien de manera puntual bien de manera transversal, han apoyado y apoyan el multilingüismo y el multiculturalismo en Europa.

Por tanto, se puede decir que el avance en políticas lingüísticas, tanto del Consejo de Europa como de la Comisión Europea, ha sido una constante en los últimos 35 años. Ha propuesto como líneas comunes: la visión de que las lenguas son necesarias para la comunicación y la movilidad (algo obvio pero que era necesario poner por escrito dentro de los documentos oficiales), el objetivo de alcanzar el multilingüismo en Europa con campañas como $1+2$ (Mother tongue plus 2), el establecimiento de que todas las lenguas europeas tengan la misma importancia, la creencia de que el entendimiento mutuo entre países se favorece con el aprendizaje de lenguas y culturas y, por último, la convicción de que la competencia lingüística lleva al desarrollo de una mejor competitividad en el sector laboral y económico. El Eurobarómetro 386 (2012) analiza las actitudes positivas hacia el multilingüismo por parte de los europeos. Señala que el $88 \%$ de los europeos piensa que conocer lenguas diferentes a la lengua madre es muy útil y el $98 \%$ considera que dominar una lengua extranjera es útil para el futuro de sus hijos. El Eurostat (2015) dice que, en 2013, 17.7 millones de alumnos de primaria de la Unión Europea $(81,7 \%)$ están estudiando al menos una lengua extranjera, incluyendo el millón (4.6\%) que están estudiando dos o más. La lengua más popular es el inglés, que está siendo estudiado por 16.7 millones de alumnos. Ambas publicaciones coinciden en concluir que existen indicadores al alza en la provisión de enseñanza en lenguas en los centros y un incremento de los niveles de competencia y de los instrumentos y materiales que apoyan la enseñanza y el aprendizaje de las mismas. Sin embargo, los logros en el desarrollo de lenguas hay que tomarlos con precaución, por ejemplo, con respecto al impacto de los programas educativos, como es el caso del Lifelong Learning Programme o de los programas del Centro Europeo de Lenguas, ya que se necesitan evidencias más claras para concluir que su alcance ha sido amplio y que su implementación no se ha reducido solo a los sectores que directamente se han visto implicados. 
Por tanto, para comprender las políticas lingüísticas de los diferentes países europeos, hay que analizar las políticas supranacionales que las enmarcan y que han sido principalmente llevadas a cabo por la Unión Europea y el Consejo de Europa. King, Byrne, Djouadj, Lo Bianco y Stoicheva (2011) realizan un análisis de las políticas lingüísticas y los nuevos contextos que han surgido. A la vez, tratan de identificar las condiciones para posibilitar el desarrollo de un marco político coherente con respecto al avance del multilingüismo. Estos autores apuntan que la articulación del multilingüismo y su tratamiento en Europa ha evolucionado desde los años 80 y ha alcanzado logros, pero también han surgido nuevas realidades y desafíos en el siglo XXI. Nuevas formas de trabajo y de comunicación, combinadas con presiones económicas y sociales hacen que el reto del multilingüismo haya ido más allá del mero marco educativo. 'Language in Europe is about more than education - it is social, economic and cultural significance' (King et al., 2011:40); y concluyen que para seguir avanzando ante estos nuevos retos, seis son las áreas que se tendrían que abordar en la nueva agenda de políticas lingüísticas: la reformulación del modelo de Lisboa hacia uno más asimétrico que propicie y se adapte a las circunstancias lingüísticas de cada región y país, la exploración de los beneficios potenciales del inglés como lengua franca, la identificación de buenas prácticas en contextos educativos multilingües, el apoyo al aprendizaje de lenguas fuera de la escuela, el posible uso multilingüe de internet y la potenciación de redes para el entendimiento global.

No obstante, lo que sí se puede afirmar es que todo ello ha ayudado de manera decisiva a crear una indudable sensibilización en la ciudadanía europea sobre la importancia del aprendizaje de lenguas y culturas para la formación de un espacio común y las lenguas se erigen, así como unas de las herramientas que poseen los ciudadanos europeos para poder tener acceso de forma igualitaria a oportunidades sociales y económicas.

\section{Aicle y las políticas lingüísticas europeas}

La importancia que la Unión Europea ha otorgado a sus políticas lingüísticas ha provocado la promoción de enfoques y metodologías, especialmente aquellas que han demostrado su eficacia para la adquisición de lenguas. Entre ellas, el Aprendizaje Integrado de Contenidos y Lenguas Extranjeras (AICLE) ha sido una de las iniciativas que más apoyo ha encontrado. En 1995, en la Resolución del Consejo de 31 de $\mathrm{Marzo}^{2}$, se menciona como uno de los enfoques innovadores que podrían ayudar a la enseñanza de lenguas dentro de los sistemas educativos europeos. De hecho, dentro del Plan de Acción (2004-2006) que la Comisión Europea lanzó después del Consejo de Europa de Barcelona, en su comunicado (Commission of the European Communities, 2003) incluía el apoyo inequívoco a la provisión de enseñanza AICLE dentro de los sistemas educativos en las propuestas para el aprendizaje de lenguas a través de la vida y se pusieron en marcha una serie de acciones para promocionarlo, como fue la financiación a través del programa Sócrates en su acción Lingua 2, su difusión a través de conferencias para responsables de políticas educativas e

2 Council Resolution of 31 March 1995 on improving and diversifying language learning and teaching within the education systems of the European Union, Official Journal C 207 of 12.08.1995. 
inspección, la publicación de estudios para aportar datos sobre la provisión (Eurydice, 2006) y la difusión de documentos para extender sus bondades dentro de los países miembro. En este mismo comunicado se expone el papel clave de las universidades para promocionar el multilingüismo a nivel social e individual, se las anima a adoptar políticas lingüísticas coherentes con la promoción de la diversidad lingüística en sus campus y a incentivar la movilidad del alumnado en países de habla extranjera. Se les aconseja también a aprovechar los recursos ya existentes (Commission of the European Communities, 2003:13)

En el simposio The Changing European Classroom: The Potential of Plurilingual Education, organizado por el Consejo de Europa en 2005, una de las principales conclusiones a las que se llegó fue a la necesidad de involucrar a alumnado y profesorado en programas AICLE para alcanzar el plurilingüismo y la pluriculturalidad en la sociedad europea. En la actualidad, la Comisión Europea hace mención especial dentro de los objetivos del programa vigente Erasmus + (2013-2020) a mejorar la enseñanza y el aprendizaje de las lenguas y promover la amplia diversidad lingüística de la Unión Europea y la sensibilización intercultural. De igual modo, en su estrategia Education and Training 2020 (ET 2020) el aprendizaje de lenguas sigue siendo una de las metas para todos los países miembro. Cabe señalar, que entre otras medidas prioritarias, la enseñanza a través del enfoque AICLE aparece como fundamental y a tener en cuenta en los diferentes niveles de enseñanza de los sistemas europeos, especialmente de los no universitarios. Julián-de-Vega (2017) apunta que a raíz de los resultados deficitarios en el aprendizaje de lenguas (la Comisión Europea (2005) aporta los siguientes datos: sólo el 36\% de los encuestados de 15 o más años de edad respondieron sentirse capaces de participar en una conversación en otro idioma distinto al materno) los países europeos empiezan a abordar la enseñanza de lenguas a través del enfoque AICLE, observándose un avance en su aprendizaje en aquellas regiones donde se ha implementado (Lorenzo, Casal y Moore, 2009). En España, será a raíz de la implantación de estos programas en las diferentes comunidades autónomas cuando las universidades empiecen a tomar las riendas y ofertar distintos programas EMI (English as Medium of Instruction) dentro de la oferta de grados para el alumnado egresado de programas AICLE en enseñanza secundaria y empezar, de esta manera, el camino al cambio dentro de las políticas lingüísticas universitarias.

\section{Políticas lingüísticas y enseñanza Plurilingüe en la educación SUPERIOR: EL CASO ESPAÑOL}

\subsection{Antecedentes}

De todo lo anteriormente dicho, resulta obligado subrayar la trayectoria que sigue la implantación de los programas AICLE en las etapas de primaria y secundaria en España porque conforman los antecedentes para entender la enseñanza AICLE en la educación universitaria. Ruíz de Zarobe y Lasagabaster (2010) señalan que en la última década el desarrollo del AICLE en el territorio español ha sido considerable, fundamentalmente debido al compromiso con las políticas europeas que promueven el plurilingüismo y la conciencia de la necesidad de aprender lenguas extranjeras. El marco legal que articula el desarrollo del AICLE en España comprende desde la Constitución Española de 1978 a leyes orgánicas 
como la Ley Orgánica reguladora del Derecho a la Educación (LODE) de 3 de julio de 1985, la Ley Orgánica de Educación (LOE) de 2006 y la Ley Orgánica 8/2013, de 9 de diciembre, para la mejora de la calidad educativa (LOMCE). El AICLE se implementa en cada una de las 17 comunidades autónomas de acuerdo a órdenes que adaptan el marco legal a las necesidades de cada comunidad; por tanto, los modelos varían de forma significativa en el territorio nacional aunque pueden dividirse en dos grandes grupos: las comunidades monolingües y las bilingües (Ruíz de Zarobe \& Lasagabaster, 2010). En las comunidades monolingües donde el español es la lengua oficial, la educación se imparte en español y en una o dos lenguas extranjeras (preferiblemente el inglés) cuando se implementa el enfoque AICLE. En las comunidades bilingües donde el español es la lengua co-oficial junto a otra lengua autonómica (euskera, catalán, gallego y valenciano) la educación se cursa en ambas en el estadio preuniversitario, además de las lenguas co-oficiales y cuando se adopta el enfoque AICLE se incorpora una lengua extranjera. El nivel de autonomía en la implementación de las leyes orgánicas de educación mencionadas arriba ha producido una gran riqueza en cuanto a planes AICLE y su praxis en el aula española (Lasagabaster, 2010), como muestra de análisis exhaustivos cabe mencionar en el caso andaluz los estudios sobre el Plan de Fomento del Plurilingüismo de la Junta de Andalucía (2004), Casal and Moore (2009), Lorenzo and Moore (2009), Lorenzo et al. (2009) y Julián-de-Vega (2017) entre otros. Queda por analizar el nivel de consecución de los objetivos curriculares a nivel nacional y su efecto en las políticas lingüísticas en la educación terciaria española.

\subsection{El camino a la acreditación lingüística en la educación universitaria}

De todo lo anterior se deduce, que en los últimos años ha habido una presión constante en la universidad española para que se adoptaran políticas lingüísticas acordes con los tiempos, ya que a sus puertas llegaban estudiantes con bagaje y niveles lingüísticos como para cursar materias en lenguas extranjeras y este desarrollo académico se veía interrumpido con las clases monolingües que proporcionaba la universidad.

La política y la planificación lingüística universitaria en el contexto español está determinada en el siglo XXI por la creación del EEES (Espacio Europeo de Educación Superior). Como se apuntaba en la visión diacrónica del hecho europeo en el apartado anterior, la evolución del multilingüismo y la publicación del MCERL han condicionado el status quo de la enseñanza de lenguas en España y por ende en la universidad española. Una de las competencias clave que se indican en el EEES es el dominio de una lengua extranjera. Si bien la universidad española ha aceptado las directrices que se marcan en el EESS, en aras de la autonomía, el desarrollo de la competencia en lengua extranjera es cuando menos heterogéneo en virtud principalmente de dos factores: el nivel de exigencia en el dominio de la lengua al terminar los grados universitarios y la forma en la que este objetivo se consigue.

El nivel de salida de los alumnos que cursan la enseñanza superior en su L2 (o L3 en el caso de los alumnos de comunidades bilingües) se sitúa en el B2 del MCER, no obstante, el Eurobarómetro 243 (European Commission, 2006) nos sitúa en el puesto 21 de 25 en conocimiento de lenguas extranjeras. Como se indicaba arriba, un problema lingüístico da lugar a políticas lingüísticas, así pues, en un primer nivel de concreción se encuentran las órdenes ministeriales que indican por ejemplo los niveles del marco que deben alcanzar los Maestros en Educación Infantil y los Maestros en Educación Primaria (órdenes ECI/3854/2007 y ECI/3857/2007), B1 para ambas titulaciones. 
Un aspecto de candente debate es precisamente cómo conseguimos en las universidades que nuestros alumnos sean competentes en al menos una lengua extranjera; dos vías complementarias aparecen en las planificaciones educativas: los centros de lenguas modernas y la implementación del enfoque integrado de lenguas y contenidos (AICLE) que algunas universidades proponen en sus titulaciones (Dafouz, 2011). Aparte de esto, hay que considerar el papel de las facultades de educación y los programas de postgrado relacionados con la enseñanza bilingüe y con la especialización en enseñanza secundaria.

La mayoría de las universidades han instaurado como mínimo el requerimiento de la acreditación del nivel B1 para poder obtener el grado, aunque algunas universidades como la de Cantabria, la de las Islas Baleares o la Politécnica de Madrid requieren un B2 para la titulación. Como indican Halbach, Lafuente, y Guerra (2013), en el caso del requerimiento del B1, sólo se está pidiendo a los alumnos que mantengan el nivel que teóricamente tenían cuando accedieron a los estudios universitarios.

\subsection{Centros de lenguas extranjeras}

Prácticamente cada universidad dispone de un centro de lenguas extranjeras, que aparecen bajo distinta denominación: centro de lenguas modernas, servicios de idiomas, instituto de idiomas, etc. El funcionamiento de estas instituciones no está regulado de forma homogénea, y en función de su año de constitución y de las necesidades lingüísticas de cada institución se han ido configurando de distinta manera. La mayoría de ellos funciona como centro examinador con acreditaciones de validez meramente interna, aunque algunos de ellos ofrecen la posibilidad de acreditación por medio de agentes externos (Cambridge, Trinity, TOEFL, etc.). El hecho de que algunos de estos centros lleven décadas funcionando hace que la diversidad en cuanto a sistemas de acreditación, programas y metodología sea notoria. En 1991 se funda la Confederación Europea de Centros de Lenguas en la Enseñanza Superior (CercleS) en Estrasburgo, 22 países europeos forman parte de ella. Con posterioridad aparece en España ACLES, Asociación de Centros de Lenguas en la Enseñanza Superior, que intenta coordinar la actividad de los centros de lenguas miembros. ACLES desarrolla de forma conjunta con la CRUE (Confederación de Rectores de las Universidades Españolas) un modelo de acreditación en idiomas con la intención de homogeneizar criterios y mecanismos de acreditación de los niveles de lenguas para:

- Acceso a titulaciones

- Desarrollo de estudios universitarios

- Programas de movilidad internacional

- Obtención de títulos de Grado y Posgrado

Con el fin de alcanzar este objetivo, la CRUE ha constituido una comisión para el análisis y estudio de la acreditación y formación en idiomas y por otro lado unas mesas lingüísticas que determinan los certificados admitidos para la acreditación de los distintos niveles lingüísticos ${ }^{3}$.

3 Véase http://www.crue.org/SitePages/Mesas-linguisticas.aspx para más detalle en relación con los certificados admitidos. 


\subsection{Facultades de Educación}

La consulta de las páginas web de las facultades de ciencias de la educación resulta esclarecedora en cuanto a la importancia que se otorga a las lenguas en la educación terciaria en particular y en el sistema de toma de decisiones en general; que la formación del profesorado de las áreas lingüísticas en educación primaria es un elemento clave de la adquisición final de los aprendientes y en definitiva de la capacidad de comunicación lingüística de la sociedad resulta hoy ya un axioma incuestionable. En relación con esta evidencia nos encontramos, por ejemplo en la página web de la Universidad de Córdoba (UCO), con que la especialidad 'Maestro en Lengua Extranjera' aparece entre las 'titulaciones extinguidas'; en la Universidad de Granada (UGR), se ofrece un grupo bilingüe dentro del grado en Educación Primaria, pero en la información administrativa encontramos: 'The Degree in Primary Education Teacher Training curriculum is the same for all groups, including the bilingual group', con lo que no existe reconocimiento oficial del esfuerzo suplementario que para el estudiante universitario ha de suponer cursar las asignaturas en una lengua extranjera. No obstante, tanto en la UGR como la Facultad de Educación de la Universidad Complutense de Madrid (UCM) y la UCO, se ofrecen menciones cualificadoras de lengua extranjera (inglés y francés) donde se cursan de forma obligatoria asignaturas de didáctica de la lengua extranjera y se exige acreditar un nivel B2 para el acceso.

La Universidad de Alicante ofrece un documento interesante sobre la situación de las menciones en los grados en Educación Primaria y Educación Infantil ${ }^{4}$. El Real Decreto $861 / 2010$, de 2 de julio, en el apartado 3 del artículo 9, 'El diseño de los títulos de Grado podrá incorporar menciones alusivas a itinerarios o intensificaciones curriculares', y éste se completa con el Real Decreto 1002/2010, de 5 de agosto, sobre expedición de títulos universitarios oficiales, en el apartado 2 del Artículo 5, referido a los Títulos de Grado, indica que: 'La denominación de estos títulos será: Graduado o Graduada en T, con Mención, en su caso, en $\mathrm{M}$, por la Universidad $\mathrm{U}$, siendo $\mathrm{T}$ la denominación específica del Grado, $\mathrm{M}$ la correspondiente a la Mención, y U la denominación de la Universidad que lo expide'.

La Orden ECI/3854/2007 y la Orden ECI/3857/2007, ambas de 27 de diciembre, por la que se establecen los requisitos para la verificación de los títulos universitarios oficiales que habiliten para el ejercicio de la profesión de Maestro en Educación Infantil y de Maestro en Educación Primaria, respectivamente, establecen que en estas enseñanzas podrán proponerse menciones cualificadoras. El artículo 2 del RD 1594/2011, sobre Especialidades docentes del Cuerpo de Maestros, establece las especialidades de lengua extranjera inglés, francés y alemán, con una atribución docente singular para cada una de dichas especialidades del Cuerpo de Maestros que resulta similar en el caso de los centros privados (RD 476/2013).

En definitiva, al amparo del proceso de adaptación de las titulaciones universitarias al Espacio Europeo de Educación Superior, de acuerdo con lo previsto en el Real Decreto 1393/2007, de 29 de octubre, por el que se establece la ordenación de las enseñanzas universitarias oficiales, modificado por el Real Decreto 861/2010, de 2 de julio, se suprimen los grados de Maestro en Lengua Extranjera quedando al albur de las facultades de educación la oferta de las menciones correspondientes.

4 Fuente: https://educacio.ua.es/es/documentos/menciones/informacion-menciones-vicent.pdf 
La oferta de las titulaciones de doble grado en Educación Primaria y Estudios Ingleses o Franceses parece abrir una puerta de esperanza a la enseñanza de la lengua extranjera en las edades tempranas, no obstante, hay que observar con detenimiento la implementación de estas ofertas educativas, velando porque los objetivos aparezcan coordinados y con una organización equilibrada entre los contenidos de formación pedagógica y los filológicos. En el caso de la Universidad de Sevilla (US), la oferta se limita al Doble Grado en Educación Primaria y Estudios Franceses.

\subsection{Postgrado}

\subsubsection{Máster de Formación del Profesorado de Enseñanza Secundaria}

En el siguiente nivel de especialización encontramos el Máster en Formación del Profesorado de Secundaria, Bachillerato, Formación Profesional y Enseñanza de Idiomas, (orden ECI/3858/2007, de 27 de diciembre), donde se exige el mismo requisito que para los grados; resulta curioso que no exista mención alguna a las especialidades del Máster que habilitan para la enseñanza de lenguas extranjeras, donde la acreditación lingüística requerida parece ser la misma. En el apartado 4.2. de dicha orden se especifican las condiciones de acceso al Máster en las que se encuentra:

Asimismo, habrá de acreditarse el dominio de una lengua extranjera equivalente al nivel B1 del Marco Común Europeo de Referencia para las Lenguas, de acuerdo con la Recomendación No R (98)6 del Comité de Ministros de Estados Miembros de 17 de octubre de 2000. (BOE núm. 312. pp 53752)

No obstante, no se hace distinción alguna para los alumnos que cursan el Máster en las especialidades de lenguas extranjeras, ni tampoco para aquellos que pudieran encaminar su carrera docente hacia la docencia en entornos bilingües; es precisamente en este punto donde podríamos contribuir a elevar el nivel instrumental de los docentes que se incorporan a los programas AICLE, que cursar el Máster de Formación del Profesorado de Enseñanza Secundaria en versión bilingüe fuera una de las posibles vías de acceso, aunque no necesariamente la única, por ejemplo los másteres en enseñanza bilingüe que se reseñan en el siguiente apartado.

\subsubsection{Másteres en enseñanza Bilingüe}

Distintas universidades ofrecen másteres en Enseñanza Bilingüe, con el objetivo común de formar profesores de enseñanza primaria y secundaria que impartan docencia en centros bilingües. En la RUCT, Registro de Universidades, Centros y Títulos, al introducir los términos de búsqueda 'bilingüe' en el apartado de Máster encontramos un total de trece másteres (dos de ellos a extinguir) adaptados al Espacio Europeo de Educación Superior (EEES) y regulados por el Real Decreto 1393/2007, de 29 de octubre, y por el Real Decreto 861/2010. Estos másteres podrían servir también como vía de acceso alternativa a los programas oficiales AICLE, en cualquier caso habría que abogar por la exigencia de requisitos formativos que vayan más allá de la mera formación instrumental en la lengua meta, la mayoría de los 
investigadores en el desarrollo de los programas AICLE aboga por invertir en la formación y la coordinación del profesorado para conseguir programas de integración de lenguas y contenidos efectivos (Pavón, Ávila, Gallego y Espejo, 2015, Delicado y Pavón Vázquez, 2015; Pavón Vázquez y Ellison, 2013; Frigols Martin, 2011; Novotná, Hadj-Moussová, y Hofmannová 2001).

\section{Conclusiones}

Para entender el cambio que se está produciendo en las políticas lingüísticas terciarias, hay que tener en cuenta y comprender las políticas lingüísticas europeas a nivel supranacional y aquellas a nivel nacional y regional donde las universidades españolas se contextualizan. En los últimos años, la Comisión Europea y el Consejo de Europa han propuesto y llevado a cabo medidas que han dado el fruto esperado. No solo la opinión pública europea ha cambiado hacia un mayor favorecimiento del aprendizaje de lenguas extranjeras, sino que la tasa de alumnado en edad escolar que está aprendiendo al menos una lengua extranjera ha ido al alza, ejerciendo una continua presión en los programas lingüísticos y de calidad de la enseñanza terciaria. El orden 'natural' en el desarrollo de una política lingüística que comienza con la identificación del problema podría, una vez puestas en marcha las medidas que se consideraron oportunas en el comienzo del proceso, dar lugar a un enfoque apreciativo (Subirana y Cooperrider, 2013) donde se identifiquen las buenas prácticas y fortalezas de los procesos multilingües iniciados y se conviertan en hábitos que permitan el crecimiento. Tanto si consideramos el marco paneuropeo como si nos circunscribimos a la situación en España, se trata de asentar ahora un proceso de búsqueda colaborativa que permita el cambio hacia una sociedad multilingüe. En el caso europeo, basta con incorporar ese análisis y universalización de buenas prácticas al marco estratégico, en el caso español, este mismo proceso habría de nutrirse de las políticas nacionales y autonómicas con resultados contrastados, de manera que se puedan adaptar a cualquiera de las situaciones lingüísticas del país.

Una de las prácticas que se ha demostrado efectiva en la promoción del aprendizaje de lenguas extranjeras es la enseñanza AICLE, que en el sistema educativo español se ha implantado en la educación primaria y secundaria en un gran número de comunidades con resultados esperanzadores, pero aún por contrastar en estudios rigurosos. La enseñanza de lenguas extranjeras en general y AICLE, en particular, en la educación terciaria parecen necesitar de vasos comunicantes con la educación no universitaria que permitan la transmisión de las buenas prácticas de un lugar a otro. A pesar de que se han introducido medidas con las nuevas normativas del EEES y con las históricamente adoptadas a través de los Centros de Lenguas, grados y postgrados, no parecen haber sido efectivas para aprender de experiencias fructíferas pasadas y nuevas relacionadas con otros entornos, posiblemente por estar condicionadas a su vez en virtud de la autonomía de acción en la mayoría de los casos. La extrapolación de buenas prácticas necesitaría la adaptación a las necesidades reales y podría facilitar enormemente el proceso de implantación de programas con enfoque AICLE. No obstante, en los últimos años, y a partir de la reflexión con respecto a las necesidades de los estudiantes para desenvolverse en un mundo globalizado, tanto la labor de la ACLES como de la CRUE parece abrir un nuevo episodio en la enseñanza de lenguas en la universidad española. 


\section{REFERENCIAS}

Casal. S. \& Moore, P. (2009) The Andalusian Bilingual Sections Scheme: Evaluation and Consultancy. International CLIL Research Journal, 1. Disponible en: http://www.icrj.eu/index. php?vol=12\&page=743, último acceso 15 septiembre, 2017.

Commission of the European Communities (2003). Communication from the Commission to the European Parliament, the Council, the European Economic and Social Committee and the Committee of the Regions. Promoting Language Learning and Linguistic Diversity: An Action Plan 2004 - 2006. Brussels. Documents COM 449 final. Disponible en: http:// eur-lex.europa.eu/LexUriServ/LexUriServ.do?uri=COM:2003:0449:FIN:en:PDF, último acceso 08 abril, 2018.

Council of Europe (2001). Common European framework of reference for languages: learning, teaching, assessment. Cambridge: Cambridge University Press.

Council of Europe (2018). Common European framework of reference for languages: learning, teaching, assessment Companion volume with new descriptors. Disponible en: https:// rm.coe.int/cefr-companion-volume-with-new-descriptors-2018/1680787989, último acceso 08 abril, 2018.

Dafouz, E. (2011). English as the medium of instruction in Spanish contexts: A look at teacher discourse. En Y. Ruíz de Zarobe, J.M. Sierra, y F. Gallardo del Puerto (Eds.) Content and foreign language integrated learning: contributions to multilingualism in European contexts. (pp. 189-210.) Frankfurt: Peter Lang.

Delicado, G., y Pavón Vázquez, V. (2015). La implantación de titulaciones bilingües en la Educación Superior: El caso de la formación didáctica del profesorado bilingüe de primaria en la universidad de Extremadura. Educación y Futuro, 32, 35-63.

European Commission (1995). White paper on education and training. towards the learning society. Brussels. Disponible en: http://europa.eu/documents/comm/white_papers/pdf/ com95_590_en.pdf, último acceso 10 febrero, 2015.

European Commission (2006). Eurobarometer 243. Europeans and their Languages. Disponible en: http://ec.europa.eu/commfrontoffice/publicopinion/archives/ebs/ebs_243_en.pdf, último acceso 10 abril, 2018.

European Commission (2012). Special Eurobarometer 386. Europeans and their Languages. Disponible en: http://ec.europa.eu/public_opinion/archives/ebs/ebs_386_en.pdf, último acceso 23 diciembre, 2017.

European Commission (2015). Eurostat. foreign language learning statistics. Disponible en: http:// ec.europa.eu/eurostat/statistics-explained/index.php/Foreign_language_learning_statistics, último acceso 04 diciembre, 2017.

European Ministers in charge of Higher Education (1999). The Bologna 1999 declaration, Disponible en: https://www.eurashe.eu/library/bologna_1999_bologna-declaration-pdf/, último acceso 08 abril, 2018.

Eurydice (2005). Key data on teaching languages at school in Europe. Brussels: Eurydice European Unit. Disponible en: http://europa.eu/legislation_summaries/education_training_youth/ lifelong_learning/ef0003_en.htm, último acceso: 4 diciembre, 2017. 
Eurydice (2006). Content and Language Integrated Learning (CLIL) at schools in Europe. Brussels: Eurydice European Unit. Disponible en: http://www.indire.it/lucabas/lkmw_file/eurydice/ CLIL_EN.pdf, último acceso 08 abril, 2018.

Frigols Martin, M.J. (2011). The European framework for clil teacher education. Language Teaching, 44(3), 401-402. http://doi.org/10.1017/S0261444811000243.

Halbach, A., Lafuente, A.L., y Guerra, J.P. (2013). La lengua inglesa en la nueva universidad española del EEES The role of the English language in post-Bologna Spanish universities, 362, 105-132. http://doi.org/10.4438/1988-592X-RE-2011-362-154

Junta de Andalucía (2004). Plan de Fomento de Plurilingüismo. Sevilla: Consejería de Educación. Documento disponible en: http://www.juntadeandalucia.es/averroes/plurilinguismo/plan.pdf, último acceso 4 septiembre, 2017.

Julián-de-Vega, C. (2017). Language policy and Teacher team coordination practices in secondary CLIL schools. European Journal of Language Policy, 9(2), 183-202.

King, L., Byrne, N., Djouadj, I., Lo Bianco, J., \& Stoicheva, M. (2011). Languages in Europe. Towards 2020. Brussels: The Languages Company.

Lasagabaster, D. (2010). CLIL in Spain: Implementation, Results and Teacher Training. Cambridge: Cambridge Scholars Publishing.

Lorenzo, F. \& Moore, P. (2009). 'European language policies in monolingual southern Europe: implementation and outcomes.' European Journal of Language Policy, 1, 121-137.

Lorenzo, F. Casal, S., \& Moore, P. (2009). The effects of content and language integrated learning in European education: key findings from the Andalusian bilingual sections evaluation project. Applied Linguistics, 31(3), 418-442.

Novotná, J., Hadj-Moussová, Z., \& Hofmannová, M. (2001). Teacher training for CLIL: competences of a CLIL teacher. Proceedings SEMT 01, 122-126. Retrieved from http://people. fjfi.cvut.cz/novotant/jarmila.novotna/Novotna-et-alSEMT01.pdf.

Orden ECI/3854/2007, de 27 de diciembre por la que se establecen los requisitos para la verificación de los títulos universitarios oficiales que habiliten para el ejercicio de la profesión de Maestro en Educación Infantil. BOE núm. 312, de 29 de diciembre de 2007, 53735-53738.

Orden ECI/3857/2007, de 27 de diciembre, por la que se establecen los requisitos para la verificación de los títulos universitarios oficiales que habiliten para el ejercicio de la profesión de Maestro en Educación Primaria. BOE núm. 312, de 29 de diciembre de 2007, 53747-53750.

Orden ECI/3858/2007, de 27 de diciembre, por la que se establecen los requisitos para la verificación de los títulos universitarios oficiales que habiliten para el ejercicio de las profesiones de Profesor de Educación Secundaria Obligatoria y Bachillerato, Formación Profesional y Enseñanzas de Idiomas. BOE núm. 312, de 29 de diciembre de 2007, 53751-53753.

Pavón Vázquez, V. \&, Ellison, M. (2013). Examining teacher roles and competences in Content and Language Integrated Learning (CLIL). Lingvarvm Arena, 4, 65-78.

Pavón Vázquez, V., Ávila López, J., Gallego Segador, A., \& Espejo Mohedano, R. (2015). Strategic and organisational considerations in planning content and language integrated learning: a study on the coordination between content and language teachers. International Journal of Bilingual Education and Bilingualism, 18(4), 409-425. 
Real Decreto 1393/2007, de 29 de octubre, por el que se establece la ordenación de las enseñanzas universitarias oficiales. BOE núm. 260, de 30 de octubre de 2007, 44037-44048.

Real Decreto 861/2010, de 2 de julio, por el que se modifica el Real Decreto 1393/2007, de 29 de octubre, por el que se establece la ordenación de las enseñanzas universitarias oficiales. BOE núm. 161, de 3 de julio de 2010, 58454-58468

Real Decreto 1002/2010, de 5 de agosto, sobre expedición de títulos universitarios oficiales. BOE núm. 190, de 6 de agosto de 2010, 68574-68609.

Subirana, M. y Cooperrider, D. (2013). Indagación Apreciativa. Un enfoque innovador para la transformación personal y de las organizaciones. Inspirémonos para diseñar nuestro futuro. Barcelona: Kairós. 